\title{
Ethical issues in NICUs: The Attitude of Greek Healthcare Professionals towards the Value of Human Life
}

Maria Daglas $^{1 *}$, Vasiliki Petousi ${ }^{2}$ and Antonios Poulios ${ }^{3}$

${ }^{1}$ Department of Midwifery, Technological Educational Institute of Athens, Greece

${ }^{2}$ Department of Sociology, University of Crete, Greece

${ }^{3}$ Department of Psychology, National Kapodestrian University, Athens, Greece

\begin{abstract}
Objective: This paper presents the first Greek empirical research on bioethical neonatal issues. The study goals were: 1) to document and measure the attitude of Greek healthcare professionals' working in NICUs towards the value of human life (intrinsic value vs. quality of life) as ethical decision making guiding principle in the provision of intensive treatment to extremely/very preterm babies and 2) to investigate the socio-cultural and other parameters which form this attitude.
\end{abstract}

Methods: Questionnaires developed for the EURONIC project and implemented in research in 11 countries were culturally adjusted to the Greek NICU context. Healthcare professionals $(n=495)$ who were employed in Greek NICUs (May 2009-May 2011) and met inclusion criteria were invited to participate. Of those 251 (98 midwives, 82 nurses and 71 doctors) completed a structured, self-administered, anonymous questionnaire (response rate $50.7 \%$ ).

Results: The reported attitude score (total sample mean attitude score $=3.09$ ) indicates that Greek healthcare professionals tend to support the intrinsic value of human life position. Gender $(p<0.05)$, the importance placed on religion $(p<0.05)$ and profession specialization $(p<0.01)$ were found to influence their attitude in statistically significant ways. Specifically, men, professionals who consider religion as being important in their life and midwives and nurses tend to be more supportive of the intrinsic value of life position. NICUs' equipment and personnel, the cost of neonatal healthcare provision, and the burden of disability on the neonate's family have not been found to influence healthcare professionals' attitude in statistically significant ways.

Conclusion: Compared to the findings from other countries in which the EURONIC research was implemented, Greek healthcare professionals appear to hold a rather vitalistic approach and follow the intrinsic value of human life position as their ethical decision-making guiding principle. Socio-cultural and professional characteristics explain ethical decision making differences among healthcare professionals.

Keywords: Healthcare; Healthcare professionals; Human life

\section{Introduction}

The birth of extremely/very preterm babies or babies with extremely low/very low birth weight continues to be a discouraging epidemiological reality with minimal (if any) important improvements in survival rates and rates of neurological and developmental problems incurring after hospital release [1-6]. This epidemiological reality however, raises serious bioethical questions for healthcare professionals responsible for the provision of neonatal intensive care $[7,8]$.

In recent years, international empirical research investigating the resolution of neonatal intensive care bioethical dilemmas has demonstrated that healthcare professionals' attitude towards the value of human life notably serves as their ethical decision making guiding principle. On the other hand, this research has documented differential patterns in the healthcare professionals' attitude [9-13].

In the context of neonatal intensive care, the bioethical dilemma relates to the limits of the provided care: should intensive care be provided to all neonates regardless of outcome or should it be withheld or withdrawn from newborns who are close to death or those who will suffer from a serious physical or mental disability in the long-term? Broadly phrased as above, the neonatal intensive care bioethical dilemma's resolution may be reached within a frame of two opposing positions: the intrinsic value of human life on the one hand and the quality of life on the other [14-17]. For the supporters of the intrinsic value of human life position, life should be protected by all means and at any cost, irrespective of its quality. Therefore, any clinical practice which acceler- ates, facilitates or simply allows the death of the patient is considered unethical and cannot be justified [14]. This view, also known as "the prolife approach", has been criticized for denying the application of limits to the care of babies with life threatening genetic disorders such as anencephaly for example [15]. On the other hand, for the supporters of the quality of life position, the value of human life is conditioned upon certain characteristics such as ability to interact with other people, ability of self-awareness, ability of self-determination. Therefore, healthcare professionals are obliged to maintain human life only in the presence of these characteristics or the potential thereof. Furthermore, since the goal of medical and nursing interventions is to respect human life albeit with certain qualitative characteristics, clinical intervention should follow evaluation of patients' (neonates' in the present study) short term (during hospitalization) and long term quality of life [16,17]. The main criticism against this position is that assessing quality of life, especially

${ }^{*}$ Corresponding author: Maria Daglas, Assistant Professor, Department of Midwifery, Technological Educational Institute of Athens, Greece, Tel: 00302109324415; E-mail: mdagla@teiath.gr

Received September 17, 2017; Accepted October 07, 2017; Published October 14, 2017

Citation: Daglas M, Petousi V, Poulios A (2017) Ethical issues in NICUs: The Attitude of Greek Healthcare Professionals towards the Value of Human Life. Health Care Current Reviews 5: 210. doi: 10.4172/2375-4273.1000210

Copyright: ( 2017 Daglas M, et al. This is an open-access article distributed unde the terms of the Creative Commons Attribution License, which permits unrestricted use, distribution, and reproduction in any medium, provided the original author and source are credited. 
in the case of newborns, is difficult, subjective, and varies, depending on the individual who performs the task [18-20].

Socio-cultural (e.g. law, religion) and ethical parameters which influence the variable attitudes of healthcare professionals towards human life [9-13], have been extensively studied in the framework of the internationally-recognized research project European Project on Parents' Information and Ethical Decision Making in Neonatal Intensive Care Units (EURONIC): Staff attitudes and opinions, which was implemented in 11 European countries in the late 90's [21]. The project, which has contributed to the development of a global discussion on ethical issues regarding newborns, was based on the opinions of healthcare professionals working in Neonatal Intensive Care Units (NICUs) $[22,23]$. Greece, did not participate in the EURONIC project and is generally lacking empirical research on bioethical issues regarding intensive care of newborns. Therefore, the attitude of Greek healthcare professionals towards the value of life of newborns who are close to death or are expected to develop a serious physical and/or mental disability remains unknown.

To address these issues, a research protocol based on the EURONIC project was recently developed and implemented for the first time in Greece as part of the first author's PhD Dissertation and under the supervision of the second author [24]. The overall goal of the research was to assess the impact of the social, cultural, religious and ethical framework on the formation of Greek NICU healthcare professionals' attitudes towards bioethical dilemmas arising from the care of extremely/very preterm babies and babies suffering from a disease. Following similar practices implemented in the countries in which the EURONIC project was conducted, project questionnaires were translated and culturally adjusted to the Greek NICU context. The EURONIC protocol was also adjusted to the specificities and needs of the research in Greece [25]. Partial findings of the above-mentioned study are reported in this paper. Thus, the aims of the present study are 1) to document and measure the attitude of Greek healthcare professionals' working in NICUs towards the value of human life (intrinsic value vs. quality of life) as ethical decision making guiding principle in the provision of intensive treatment to extremely/very preterm babies and 2) to investigate the socio-cultural and other parameters which form this attitude.

\section{Materials and Methods}

\section{Sample}

Following the EURONIC protocol as implemented in small countries, all healthcare professionals working in NICUs of Greek public hospitals were invited to participate in the study. Data collection took place from May 2009 until March 2011 [21]. Of the total 18 Greek NICUs, 17 satisfied the EURONIC project inclusion criteria and agreed to participate. All full-time doctors, midwives and nurses employed in the selected NICUs $(\mathrm{n}=495)$ were invited to participate; of these, 251 healthcare professionals (71 doctors, 98 midwives, 82 nurses) responded positively (response rate $50.7 \%$ ) [21,25].

\section{Questionnaires}

In our study we implemented three structured questionnaires originally developed in the EURONIC project which were modified and adjusted to the Greek context: a) the "Questionnaire for Medical Staff', b) the "Questionnaire for Nursing Staff' and c) the "Unit Description Questionnaire". The a) and b) above, original EURONIC project questionnaires, were translated from English into Greek and back translated to English following best practices and guidelines of the American Association of Orthopaedic Surgeons Outcomes Committee
[26,27]. They were then pilot tested and adjusted accordingly. In the context of our research, EURONIC questionnaire c) above, was not possible to be implemented as originally designed. A different version was developed for the purposes of the present study. The translation and cultural adaptation of the EURONIC project's questionnaires in the context of our research are analytically described in another article [25].

For the purposes of the current article we analysed healthcare professionals' responses to 12 attitudinal statements, which were included in the second part of the questionnaires for medical and nursing staff. The content of the original statements which we adapted for our research was decided on the basis of the international literature and in-depth qualitative interviews among healthcare professions in different countries $[9,21,28]$. It was further evaluated by a team of experts of different scientific and cultural backgrounds [9-11,13,29,30].

Based on research in 10 different countries, it was found that 7 of the 12 statements have a higher inter-correlation and thus, better reliability (Cronbach $\alpha=0,71$ ) [9]. Consequently, following previous research, we also used these 7 statements to estimate healthcare professionals' attitude towards the value of life.

Responses to these statements are measured on the five-point Likert type scale (from "strongly agree" to "strongly disagree"). Opinions can vary from full agreement with the position of the absolute value of human life ("The pro-life approach") advocating thus, that the life of a newborn should be maintained regardless of outcome and cost, to full disagreement with this position advocating thus, that the value of human life and the justified interventions should be decided on the basis of the quality of human life ("The quality-of-life approach") [9]. Based on these scaled answers, the attitude score was calculated. The attitude score represents the sum of each participant's answers to 7 out of the 12 statements. Score values range from 0 (attitude in favour of "pro-life approach") to 10 (attitude in favour of the "quality-of-life approach"). Higher attitude scores indicate support towards the quality of life position; lower attitude scores indicate support towards the intrinsic value of life position [9].

\section{Research ethics}

Throughout the study, special attention was given to respecting the code of conduct of research and in particular the principles of informed consent, and the protection of respondent's identity and confidentiality [25]. Written permission to carry out the research was granted by the Board of Directors and Ethics Committee of all participating hospitals. Additional, written consent and permission was granted by the Directors of all participating NICUs. Written informed consent was sought and received by each participant. Questionnaires contained no personal identifiers. To further safeguard against respondent's identification, questionnaires were self-administered. Each returned questionnaire was assigned a serial number (not linked in any way to respondent's identity) and a code indicative of the NICU.

\section{Statistical analysis}

Data analysis was performed using the SPSS software. The attitude of healthcare professionals towards the value of human life was set as the dependent variable for all analyses. The attitude of healthcare professionals towards the value of human life was assessed by the attitude score, the sum of each participant's answers to the 7 attitudinal statements measured on a five-point Likert scale as described above. The 7 attitudinal statements defining the attitude score were found to have a high degree of intercorrelation (Cronbach $\alpha=0.70$ ) and thus high reliability. The validity of the used attitudinal statements is further 
consolidated by the fact that it has been repeatedly and successfully used in numerous studies [9-11,13].

As independent variables in the single-factor analyses, we selected parameters that, according to the international literature, are related to the attitude of healthcare professionals towards human life and determine the framework of ethical decision-making regarding treatment of newborns. Specifically, independent variables consisted in participants' socio-demographic, cultural and professional characteristics such as gender, age, children, importance of role of religion, professional title, length of experience in NICUs, as well as framework (i.e., structural and functional condition as expressed by number of hospitalized newborns, equipment, personnel), location, equipment and personnel of NICUs.

At the single-factor level, for variables consisted of two categories we compared means with an independent samples t-test. In the case of variables consisted of more than two categories, we performed OneWay Analysis of Variance (ANOVA). In ANOVA analyses, the attitude score was the dependent variable and the different socio-cultural, demographic and professional parameters were the independent variables. In order to control for Type I error, multiple comparisons in these analyses were performed using Scheffés and Tamhane's tests, when the homogeneity hypothesis was discarded after performing the Levéne's test or when there was an important size difference among the different groups. Pearson's $r$ correlation was used for the calculation of correlation between numerical variables.

Besides single-factor analyses, a statistical prediction model was built to identify and evaluate the effect of the variables associated with the healthcare professionals' attitude score. Regression analysis (enter method) was used in this model, since the dependent variable (the attitude score) was numerical. In the regression model, only variables found to be statistically significant in the single-factor analysis were introduced.

\section{Results}

The sociodemographic and professional characteristics of the 251 participants are presented in Table 1. Participants were mostly women (91.1\%); aged 30-50 years old (71.5\%) and half of them had children (52.7\%). Almost all are Christian Orthodox (98.6\%) and tend to consider the role of religion in their life from fairly to extremely important $(72.9 \%)$. As regards the professional characteristics of the study population, it comprised 39\% midwives, $32.7 \%$ nurses and 28.3\% medical doctors. Almost half of the sample works in universityaffiliated hospitals (54.6\%), over half of the sample in provincial hospitals (56.2\%), while the majority of sample (78.5\%) has less than 15 years professional experience in NICU. Participants worked in 17 Greek NICUs, the characteristics of which regarding equipment and human resources are presented in Table 2. On average, these NICUs had a capacity of 30.14 nursing neonates, 9.5 mechanical ventilation devices (respirators) and a total of 28.5 employees (10.31 doctors, 21.2 midwives and nurses) with professional experience in NICU of 8.95 years (Table 2).

Table 3 shows the proportion of healthcare professionals responding "agree" or "strongly agree" to the 12 statements of the questionnaires exploring their attitudes. Highlighted in Table 3 are respondents' answers to the 7 statements which constitute their attitude score. Overall, the average of the attitude score for the total sample in our study is 3.09. Differences however, are noted in the average attitude score for the different groups of healthcare professions included in the

\begin{tabular}{|c|c|c|}
\hline $\begin{array}{l}\text { Demographic } \\
\text { characteristics }\end{array}$ & $\mathbf{n}$ & $\%$ \\
\hline \multicolumn{3}{|l|}{ Age } \\
\hline$<30$ years & 46 & 21.3 \\
\hline $30-39$ years & 83 & 37.9 \\
\hline $40-49$ years & 70 & 33.6 \\
\hline 50 years and over & 14 & 7.2 \\
\hline \multicolumn{3}{|l|}{ Gender } \\
\hline Male & 19 & 8.9 \\
\hline Female & 200 & 91.1 \\
\hline \multicolumn{3}{|l|}{ Having children } \\
\hline Yes & 107 & 52.7 \\
\hline No & 96 & 47.3 \\
\hline \multicolumn{3}{|c|}{ Religious background } \\
\hline Christian Orthodox & 218 & 98.6 \\
\hline None & 3 & 1.4 \\
\hline \multicolumn{3}{|c|}{ Importance of religion } \\
\hline Extremely important & 71 & 30.1 \\
\hline Enough important & 101 & 42.8 \\
\hline Not very important & 35 & 14.8 \\
\hline Not at all important & 21 & 8.9 \\
\hline No answer & 8 & 3.4 \\
\hline \multicolumn{3}{|l|}{$\begin{array}{l}\text { Professional } \\
\text { characteristics }\end{array}$} \\
\hline \multicolumn{3}{|l|}{ Professional title } \\
\hline Doctor & 71 & 28.3 \\
\hline Midwife & 98 & 39 \\
\hline Nurse & 82 & 32.7 \\
\hline \multicolumn{3}{|l|}{ Institution } \\
\hline University & 114 & 54.6 \\
\hline Public & 99 & 45.4 \\
\hline \multicolumn{3}{|l|}{ Hospital location } \\
\hline Urban & 101 & 43.8 \\
\hline Provincial & 112 & 56.2 \\
\hline \multicolumn{3}{|l|}{ Experience at NICU } \\
\hline$<6$ years & 107 & 47.6 \\
\hline $6-15$ years & 67 & 30.9 \\
\hline$>15$ years & 47 & 21.5 \\
\hline
\end{tabular}

Table 1: Socio-demographic and professional characteristics of participants.

\begin{tabular}{|l|c|c|c|c|}
\hline & Min & Max & Mean & Std. Deviation \\
\hline Total number of nursing neonates & 7 & 45 & 30.14 & 11.3 \\
\hline Total number of mechanical respirators & 2 & 17 & 9.5 & 4.26 \\
\hline $\begin{array}{l}\text { Total number of employees (doctors, } \\
\text { midwives and nurses) }\end{array}$ & 14 & 43 & 28.5 & 8.58 \\
\hline Total number of doctors & 3 & 15 & 10.31 & 3.23 \\
\hline Total number of midwives and nurses & 10 & 30 & 21.2 & 6.45 \\
\hline Professional experience (years) & 0 & 30 & 8.95 & 7.4 \\
\hline
\end{tabular}

Table 2: Equipment and personnel of the 17 Greek NICUs included in the study.

sample. Specifically, the average attitude score for medical doctors is 3.26 (SE 0.59), for midwives 3 (SE 0.52) and for nurses 3.04 (SE 0.56). Based on the above, it can be argued that Greek healthcare professionals tend to assign intrinsic value to human life. It should follow, thus, that Greek NICU professionals would support continuance of life regardless of the qualitative result or the required healthcare cost. 
'Agree' or 'Strongly agree' $\%$ (f)

Withholding intensive care without simultaneously taking active measures to end the neonate's life is dangerous because it makes it more likely for the neonate to be severely disabled if he/she survives

$57.2 \%(135)$

* Every neonate should be given the maximum amount of intensive care irrespective of outcome, because the clinical experience acquired will benefit other patients in the future

From an ethical point of view, there is no difference between withholding and withdrawing of intensive care

From an ethical point of view, there is no difference between withdrawal of intensive care and administration of drugs with the purpose of ending life

* Limiting intensive care, even if only in extremely selected situations, is a 'slippery slope' that will lead to abuses

* There is no room for ethical decisions when the law does not allow any limitations of treatment

* Even with severe physical disability, some life is always better than no life at all

* Because human life is sacred, everything possible should be done to ensure a neonate's survival, however severe the prognosis

* The burden that a disabled child will represent for the family is not so relevant when making ethical decisions for that neonate Intensive care is a 'slippery slope' likely to lead to therapeutic aggressiveness

The increasing costs of healthcare for preterm newborns and disabled children do not allow us to treat each patient regardless of outcome

* Even with severe mental disability, some life is always better than no life at all

$48 \%(118)$

$47.8 \%(116)$

$40.6 \%$ (99)

$38 \%(90)$

$37.5 \%$ (91)

$36.8 \%(91)$

$35.1 \%(86)$

$26.7 \%(65)$

$24 \%$ (57)

$23.7 \%(58)$

$23.1 \%(57)$

"In bold, the 7 statements defining the attitude score on the value of human life

Table 3: Healthcare professionals' agreement to 12 statements assessing the attitude towards the value of human life.

Looking further into the individual statements constituting the attitude score the following can be observed. Nearly half of the participants (48\%) support provision of treatment for every neonate, irrespective of outcome, on the basis that "the clinical experience acquired will benefit other patients in the future", making thus and reference to the betterment of science for the benefit of future patients. Furthermore, $38 \%$ of our sample considers that "limiting intensive case even if only in extremely selected situations as a 'slippery slope' that will lead to abuses" while a considerable proportion of Greek healthcare professionals appears to give restrictive competence to legal provisions since $37.5 \%$ of them agree or strongly agree that "there is no room for ethical decision when the law does not allow any limitations of treatment". Explicit support to the inherit value of life and the provision of all possible treatment regardless of prognosis is provided by $35.1 \%$ of our sample while $26.7 \%$ of them state that the burden a disabled child may bring to the family is not a parameter of consideration in ethical decision making. Nevertheless, based on our findings, healthcare professionals tend to somewhat differentially assess physical and mental disability to the extent that $26.8 \%$ of our sample argues that "even with severe physical disability, some life is better than no life at all" in comparison to the $23.1 \%$ of our sample who hold a similar view with regards to mental disability.

Although not included in the statements which form the attitude score, some of our participants' responses corroborate the above findings and merit special attention. Specifically, almost half participants $(47.8 \%)$ don't make an ethical distinction between withholding and withdrawing intensive care while $40.6 \%$ do not make an ethical distinction between withdrawing intensive care and ending a neonate's life. Nevertheless, over half of our sample (57.2\%) states that "withholding intensive care without simultaneously taking active measures to end the neonate's life is dangerous because it makes it more likely for the neonate to be severely disabled if he/she survives". Finally, only $1 / 4(23.7 \%)$ of the participants considers the cost of healthcare for preterm newborns to be a restrictive factor in the provision of care.

To further explore the factors that affect the attitude of Greek healthcare professionals towards the value of human life, we first estimated potential correlations between this attitude and different factors independently, using the Pearson's correlation statistic. These factors were gender, age, parenthood, importance of religion, professional title, professional experience in NICUs, location of the

\begin{tabular}{|c|c|c|}
\hline Variables & Attitude Score $(95 \% \mathrm{Cl})$ & $\mathrm{P}$ value *** \\
\hline Sex & & 0.863 \\
\hline Male & $2.9(2.8-3.1)^{*}$ & \\
\hline Female & $3.1(2.9-3.2)$ & \\
\hline Age & & 0.64 \\
\hline$<30$ years & $2.9(2.7-3.1)^{\star *}$ & \\
\hline 30-39 years & $3.2(3-3.3)$ & \\
\hline 40-49 years & $3.2(3.1-3.4)$ & \\
\hline 50 years and more & $3.1(2.7-3.5)$ & \\
\hline Having children & & 0.19 \\
\hline Yes & $3.1(2.9-3.2)^{*}$ & \\
\hline No & $3(2.9-3.2)$ & \\
\hline Importance of religion & & 0.033 \\
\hline Important & $3.1(2.9-3.2)^{\star *}$ & \\
\hline Not important & $3.3(3.1-3.5)$ & \\
\hline Professional title & & 0.057 \\
\hline Doctors & $3.3(3.1-3.4)^{\star *}$ & \\
\hline Midwives & $3(2.9-3.1)$ & \\
\hline Nurses & $3(2.9-3.2)$ & \\
\hline Experience at NICU & & 0.949 \\
\hline$<6$ years & $3.1(3-3.2)^{\star \star}$ & \\
\hline $6-15$ years & $3.1(3-3.3)$ & \\
\hline$>15$ years & $3.1(2.9-3.3)$ & \\
\hline Institution & & 0.811 \\
\hline University & $3(2.9-3.2)^{\star}$ & \\
\hline Public & $3.1(3-3.2)$ & \\
\hline Hospital location & & 0.724 \\
\hline Urban & $3.1(2.9-3.3)^{\star}$ & \\
\hline Rural & $3.1(3-3.3)$ & \\
\hline
\end{tabular}

* Mean values of attitude score was the result of t-test analyses for independent samples

${ }^{* *}$ Mean values of attitude score was the result of performance of ANOVA analyses According to Rebagliato et al. [9], lower values reflect the "prolife" attitude and higher values the "quality-of-life" attitude.

${ }_{* \star *} P$ value refers to the statistical significance of the association between a given variable and the attitude score. Significant $P$ values are marked in bold

Table 4: Univariate association analysis of attitude score with different variables.

hospital and framework. No significant correlation was found in this analysis (data not shown). Next, we proceeded with one-way ANOVA (Table 4). Interestingly, the only factor that was shown to significantly impact respondents' attitude towards the value of human life is the 


\begin{tabular}{|l|c|c|c|}
\hline Prediction variables & B & SE B & beta \\
\hline Gender & $\mathbf{2 . 9 8}$ & $\mathbf{1 . 2 7}$ & $\mathbf{0 . 1 8}^{*}$ \\
\hline Age & 0.27 & 0.99 & 0.03 \\
\hline Having children & -0.46 & 0.80 & -0.05 \\
\hline Importance of religion & $\mathbf{- 2 . 0 6}$ & $\mathbf{0 . 8 1}$ & $\mathbf{- 0 . 1 8 ^ { * }}$ \\
\hline Experience at NICU & 0.07 & 0.06 & 0.10 \\
\hline Professional title & $\mathbf{- 2 . 3 3}$ & $\mathbf{0 . 8 2}$ & $\mathbf{- 0 . 2 2}$ \\
\hline Institution & -0.23 & 0.76 & -0.02 \\
\hline Hospital location & 0.41 & 0.75 & 0.04 \\
\hline
\end{tabular}

Note. ${ }^{*} \mathrm{p}<0.05,{ }^{* *} \mathrm{p}<0.01$

Dependent variable: the healthcare professionals' attitude score on the value of human life (Method enter). $R^{2}=0.04, F(9,198)=2.03, p<0.05$

Table 5: Prediction variables of the regression analysis model for the attitude score.

importance of religion ( $\mathrm{p}=0.033$ ): Greek healthcare professionals who stated that religion plays an important role in their lives had a lower attitude score. They tended thus, to support the inherent value of life position, more so than their counterparts who considered the role of religion as less/not important.

As a next step, a regression analysis model was built, in order to identify the set of responders' characteristics that significantly associate with the "attitude score", and which might explain its variability among Greek healthcare professionals. The same variables considered in the simple-factor analyses were set as prediction variables. As shown in Table 5, three (out of a total of eight) factors significantly contributed to the prediction of the "attitude score". These are: a) gender $(\beta=0.18$, $\mathrm{t}=2.34, \mathrm{p}<0.05), \mathrm{b})$ importance of religion $(\beta=-0.18, \mathrm{t}=-2.56, \mathrm{p}<0.05)$ and $c)$ professional title $(\beta=-0.22, t=-2.85, p<0.01)$. Specifically, higher attitude scores" were significantly associated with female healthcare professionals, individuals who considered the role of religion as not important in their lives, and medical doctors. Consequently, the above groups tend to subscribe to the quality of human life argument as guiding principle in their ethical decision-making.

\section{Discussion and Conclusion}

The aim of this study was to document and assess the attitude of Greek healthcare professionals working in NICUs towards two opposing principles, the intrinsic value of human life and the quality of life. In addition, the study aimed to explore the socio-cultural factors that determine this attitude. Our study is the first to collect and present data from Greece concerning the issue. Greece does not often participate in discussions on bioethical issues regarding newborns, and has neither empirical data nor relevant guidelines available for healthcare professionals. Thus, the present research has the potential to positively contribute to the development of the relevant bioethical discourse and debate. Detailing, on the one hand, the prevailing attitude of healthcare professionals towards the value of human life and on the other hand, the factors affecting the formation of this attitude, is necessary in order to ascertain whether and to what extent this attitude is linked to the clinical behaviour of healthcare professionals and especially to the ethical decision-making on withholding, withdrawing or actively terminating the life of a newborn.

The research tools employed here were based on those developed in the context of the European EURONIC project and are, thus, part of the largest-to-date research protocol implemented and applied worldwide to tackle this issue [26]. Greek healthcare professionals were invited to express their opinion taking into account ethical and legal restrictions, the cost of healthcare services, the benefit of science and the burden that a child's disability entails for the family. The attitude towards the value of human life was explored using the attitude scale of Rebagliato et al (2000) [9]. The application of a common tool in this study is of high value, since it allows data comparison across countries that participated in the EURONIC project. As expected, the study is not devoid of limitations, which mainly relate to the relatively small sample size which is, however, depended on the target population, the sampling method and the inclusion criteria. That is, to a large extent, the sample size is associated with the number of NICUs in the country [25].

Based on the attitude score, Greek healthcare professionals working in NICUs tend to support the argument of the intrinsic value of human life. As a matter of fact, the average attitude score recorded for the entire sample and the different groups in our sample (3.09 for the entire sample, 3.26 for medical doctors, 3 for midwives and 3.04 for nurses) is the lowest from all countries in which the EURONIC project was carried out $[9-11,13]$. Similar, albeit higher, scores were previously recorded in Hungary (score 5.2), the Baltic countries (Estonia 4.9 and Lithuania 5.5), Italy (5.7) and Ireland (5.8) $[9,10]$. On the contrary, in countries like the United Kingdom, the Netherlands, Sweden and the United Stations of America, the prevailing attitude is the one supporting the principle of quality of life $[9,13]$.

In accordance with the low attitude score the proportion of Greek healthcare professionals who accept the inherent value of human life and thus, support maintenance and continuance of life in case of unfavourable prognosis is relatively high (35.1\%) compared to equivalent proportions within the EURONIC project. This proportion is similar to that of Italian healthcare professionals (33\%) [9] Furthermore, the percentage of Greeks who agree with the statement "life is always preferable to death even in the case of serious mental disability" (23.1\%) is similar to that of their Italian (23\%) and Estonian colleagues (22\%), while in the case of physical disability the respective percentage of Greeks who agree (36.8\%) is similar to that of the Irish (37\%), Swedish (36\%) and Lithuanians (36\%) $[9,10]$. The degree of consent of healthcare professionals with the above statement is lower in the case of mental disability not only in Greece, but worldwide [9-13]. It can be argued thus, that the presence or potential of mental disability is considered as exerting more negative impact on the quality of life than physical disability.

It is important to note that the following three statements received the highest percentage of agreement from healthcare professionals in our sample: a) "withholding intensive care without taking measures to end a neonate's life is dangerous because of the potential for severe disabilities", b) "there is no moral distinction between withholding and withdrawing intensive care" and c) "there is no moral distinction between withholding intensive case and actively ending the life of a neonate." As noted above, these statements are not included among those defining the attitude score. Nevertheless, the high acceptance rate they receive among Greek healthcare professionals indicate that when drawing conclusions over the guiding principle (intrinsic value of life $v s$. quality of life) in ethical decision-making, responses to the full questionnaires and the specificities of each country need to be taken into account.

According to the EURONIC project, an important prediction factor of the attitude of healthcare professionals towards the value of human life (intrinsic $v s$. quality) is the country itself [9]. This result reflects the impact of cultural and social parameters on the creation and adoption of a value system. Among the different factors considered in this study as potentially affecting the attitude of Greek healthcare professionals, religion stands out as the most important. This parameter was identified 
as statistically significant in both univariate and multivariate analyses (regression analysis). The overwhelming majority of Greeks are Christian Orthodox, and the population shows a high attachment to its religious background (in this study, 72.9\%). The religious background was highlighted as an important parameter for the attitude score in other studies, too. Notably, these studies concern countries that traditionally have a strong religious component such as Italy, Spain, Ireland, Turkey and Central America [9-12]. Additional factors significantly associated with the attitude score in the regression analysis were, similarly to reports from the EURONIC project [9], gender and the professional title at the NICU (i.e., doctors versus midwives versus nurses). The tendency to support the intrinsic value of life view is more prominent in men, midwives and nurses and individuals who consider the role of religion as important in their lives. Additional analyses and research would be necessary to fully reveal the reasons for this distribution.

A number of additional parameters identified in the international literature as affecting the attitude of healthcare professionals towards human life such as, healthcare cost and the burden a disabled child may represent for the family, were not found to impact Greek hearth care professionals' ethical decision making in statistically significant ways. Specifically, the family's burden of care for a disabled child has been revealed as a crucial factor in ethical decision making concerning neonatal intensive care in all the countries in which the EURONIC project was carried out $[9-11,13]$. On the contrary, in Greece, such consideration is important to only $26.7 \%$ of our sample. Similarly, neonatal intensive care health costs frequently reflecting the reality of limited healthcare resources [12] as well as other specificities of the healthcare system were found to be decisive factors in professionals' ethical decision making in various countries and at varying patterns [9$11,31,32]$. In Greece, such matters appear to be taken into consideration by only $23.7 \%$ of our sample.

Finally, national legal regulation and the potential of legal action against them is revealed in some research to affect healthcare professionals' decision making [33]. Other research, however, finds that legal parameters do not impact upon such decisions [32,34] pointing thus, not only to inconclusiveness about the impact of regulations but also to the complexity of the relationship and interdependencies between the loci of ethics and law. The complexity of the above relationship is evident in our study. First, it needs to be noted that a specified legal and regulatory framework delineating clinical practices is missing, apart from professional codes of ethics and general guidelines, with, admittedly, minimal imperative mandate. On the other hand, although multivariate analysis did not reveal the regulatory framework to impact healthcare professionals' ethics decision making in statistically significant ways, a proportion $(37.5 \%)$ of our sample tended to agree that a legal framework would be critically affecting neonatal intensive care decision making To conclude, the present study represents a valuable attempt to both report on, and sensitize Greek healthcare professionals dealing with bioethical dilemmas concerning provision of intensive healthcare to extremely/very preterm babies. We report a vitalistic attitude towards the value of neonatal life, which exceeds previously reported similar attitudes among European countries. Our study further identified the most important sociocultural factors which form the attitudes of Greek healthcare professionals either in support of the intrinsic value of human life or the quality of life approach as ethical decision making guiding principles $[9,34,35]$. These were the importance of religion, the professional title and the gender of respondents, but not the conditions in NICUs (equipment and personnel), the cost of neonatal healthcare and the burden that a disability entails for the family. In light of the country's recent economic turmoil and its detrimental negative impact on the healthcare system, it may be of interest to repeat a similar study and check whether the impact of the latter parameters (NICU conditions, healthcare cost and burden to the family) continue to be statistically non-significant. Furthermore, in order to gain a more in-depth understanding of the way social and cultural parameters affect the formation of opinions on this complex bioethical dilemma, future studies, which will include qualitative research methods, follow-up questionnaires to increase the sample size, and systematic comparisons across countries on specific issues of complex nature, are expected.

\section{Acknowledgement}

We are very grateful to Professor Maria Cuttini for giving us the permission to use the questionnaires of the European Concerted Action project EURONIC on Parents' information and ethical decision-making in neonatal intensive care units: staff attitudes and opinion funded by the European Commission (Contract No BMH1-CT93-1242, project coordinator M. Cuttini).

\section{References}

1. Moore T, Hennessy EM, Myles J, Johnson SJ, Draper ES, et al. (2012) Neurological and developmental outcome in extremely preterm children born in England in 1995 and 2006: The EPICure studies. BMJ 34: 7961.

2. Marlow N, Bennett C, Draper ES, Hennessy EM, Morgan AS, et al. (2014) Perinatal outcomes for extremely preterm babies in relation to place of birth in England: The EPICure 2 study. Arch Dis Child Fetal Neonatal Ed 99: 181-188.

3. Msall ME (2007) The limits of viability and the uncertainty of neuroprotection Challenges in optimizing outcomes in extreme prematurity. Pediatrics 119: 158160

4. Tommiska V, Heinonen K, Lehtonen L, Renlund M, Saarela T, et al. (2007) No improvement in outcome of nationwide extremely low birth weight infant populations between 1996-1997 and 1999-2000. Pediatrics 119: 29-36.

5. Glass HC, Costarino AT, Stayer SA, Brett CM, Cladis F, et al. (2015) Outcomes for extremely premature infants. Anesth Analg 120: 1337-1351.

6. Grisaru-Granovsky S, Reichman B, Lerner-Geva L, Boyko V, Hammerman C, et al (2014) Population-based trends in mortality and neonatal morbidities among singleton, very preterm, very low birth weight infants over 16 years. Early Hum Dev 90: 821-827.

7. Strandås M, Fredriksen ST (2015) Ethical challenges in neonatal intensive care nursing. Nurs Ethics 22: 901-912.

8. Prentice T, Janvier A, Gillam L, Davis PG (2016) Moral distress within neonata and paediatric intensive care units: A systematic review. Arch Dis Child 101 701-708.

9. Rebagliato M, Cuttini M, Broggin L, Berbik I, de Vonderweid U, et al. (2000) Neonatal end-of-life decision making: Physicians' attitudes and relationship with self-reported practices in 10 European countries. JAMA 284: 2451-2459.

10. Samaan MC, Cuttini M, Casotto V, Ryan CA (2008) Doctors' and nurses' attitudes towards neonatal ethical decision making in Ireland. Arch Dis Child Fetal Neonatal Ed 93: 217-21.

11. Bilgen H, TopuzoAYlu A, KuAYAGu K, Altuncu E, Ozek E (2009) End-of-life decisions in the newborn period: Attitudes and practices of doctors and nurses. Turk J Pediatr 51: 248-256

12. MartinezAM, Mathes ED, Foster-Rosales AF, Partridge JC (2009) Obstetricians' attitudes and practices of life support for extremely premature low birth weight infants in El Salvador. J Neonatal Perinatal Med 2: 49-56.

13. Donohue PK, Boss RD, Aucott SW, Keene EA, Teague P (2010) The impact of neonatologists' religiosity and spirituality on health care delivery for high-risk neonates. J Palliat Med 13: 1219-1224.

14. Tripp J, McGregor D (2006) Withholding and withdrawing of life sustaining treatment in the new-born. Arch Dis Child Fetal Neonatal Ed 91: F67-71.

15. Pinter $A B$ (2008) End-of-life decision before and after birth: Changing ethical considerations. J Pediatr Surg 43: 430-436.

16. Kluge $\mathrm{EH}$ (2006) Hope in the neonatal intensive care nursery: Values, ethics and the injury of continued existence. Med Gen Med 27: 74

17. Wilkinson DJ (2011)A life worth giving? The threshold for permissible withdrawa of life support from disabled new-born infants. Am J Bioeth 11: 20-32. 
Citation: Daglas M, Petousi V, Poulios A (2017) Ethical issues in NICUs: The Attitude of Greek Healthcare Professionals towards the Value of Human Life. Health Care Current Reviews 5: 210. doi: 10.4172/2375-4273.1000210

Page 7 of 7

18. Haward MF, Kirshenbaum NW, Campbell DE (2011) Care at the edge of viability: Medical and ethical issues. Clin Perinatol 38: 471-492.

19. Kluge EH (2009) Quality-of-life considerations in substitute decision-making for severely disabled neonates: the problem of developing awareness. Theor Med Bioeth 30: 351-366.

20. Weir M1, Evans M, Coughlin K (2011) Ethical decision making in the resuscitation of extremely premature infants: The health care professional's perspective. J Obstet Gynaecol Can 33: 49-56.

21. Cuttini M, Kaminski M, Saracci R, de Vonderweid U (1997) The EURONIC project: A European concerted action on information to parents and ethical decision-making in neonatal intensive care. Paediatr Perinat Epidemiol 11 461-474.

22. Pierce C, Kenny M, Peters M, Mok Q, Petros A (2000) End-of-life decisions for new-born infants. Lancet 356: 946.

23. Walther FJ (2005) Withholding treatment, withdrawing treatment and palliative care in the neonatal intensive care unit. Early Hum Dev 81: 965-972.

24. Daglas M (2015) Bioethical decisions regarding the care of infants during perinatal period: Views of healthcare professionals in Greece.

25. Daglas M, Petousi V (2016) Ethical decision making in neonatal intensive care: Adaptation of EURONIC research protocol in research in Greece. Clin Exp Obstet Gynecol.

26. Beaton DE, Bombardier C, Guillemin F, Ferraz MB (2000) Guidelines for the process of cross-cultural adaptation of self-report measures. Spine (Phila $\mathrm{Pa}$ 1976) 25: 3186-3191

27. Acquadro C, Conway K, Hareendran A, Aaronson N (2008) European regulatory issues and quality of life assessment (ERIQA) group literature review of methods to translate health-related quality of life questionnaires for use in multinational clinical trials. Value Health 11: 509-521.

28. Garel M, Gosme-Séguret S, Kaminski M, Cuttini M (1997) Ethical decisions making in neonatal intensive care. Survey among nursing staff in 2 French centers. Arch Pediatr 4: 662-670

29. Cuttini M, Casotto V, Kaminski M, de Beaufort I, Berbik I, et al (2004) Should euthanasia be legal? An international survey of neonatal intensive care units staff. Arch Dis Child Fetal Neonatal Ed 89: 19-24.

30. Garel M, Caeymaex L, Goffinet F, Cuttini M, Kaminski M (2011) Ethically complex decisions in the neonatal intensive care unit: Impact of the new French legislation on attitudes and practices of physicians and nurses. J Med Ethics 37: 240-243.

31. Gallagher K, Martin J, Keller M, Marlow N (2014) European variation in decision-making and parental involvement during preterm birth. Arch Dis Child Fetal Neonatal Ed 99: 245-249.

32. Verhagen AAE, van der Hoeven MAH, van Meerveld RC, Sauer PJJ (2007) Physician medical decision-making at the end of life in new-borns: Insight into implementation at 2 Dutch Centers. Pediatrics 120: e20-28.

33. Swinton CH, Lantos JD (2010) Current empirical research in neonatal bioethics Acta Paediatr 99: 1773-1781.

34. Sauer PJ, Dorscheidt JH, Verhagen AA, Hubben JH (2013) Medical practice and legal background of decisions for severely ill new-born infants: Viewpoints from seven European countries. Acta Paediatr 102: 57-63.

35. Cuttini M, Casotto V, de Vonderweid U, Garel M, Kollée LA, et al. (2009) Neonata end-of-life decisions and bioethical perspectives. Early Hum Dev 85: S21-25. 